\title{
ORIGINAL
}

\section{BROTE DE HEPATITIS A EN GALICIA DURANTE 2016-2018}

\author{
Nuria Suárez Gaiche (1), María Jesús Purriños-Hermida (2) y Anxela Pousa Ortega (2) \\ (1) Servicio de medicina preventiva y salud pública. Complexo Hospitalario Universitario de Santiago de Compostela. A Coruña. España. \\ (2) Servizo de epidemioloxía. Dirección xeral de Saúde Pública. Consellería de Sanidade. Santiago de Compostela. A Coruña. España. \\ Conflictos de interés: María Jesús Purriños-Hermida tiene un contrato puntual de consultoría con Shionogi SL, para la realización de un \\ decálogo sobre la importancia de las resistencias antimicrobianas. El resto de las autoras declaran no tener ningún conflicto de interés.
}

\section{RESUMEN}

Fundamentos: En Galicia, la incidencia (I) de hepatitis A (HA) es baja y la susceptibilidad es del $51 \%$ en adultos (18-64 años). Entre 2016 y 2018 se incrementaron los casos, fundamentalmente en hombres. El objetivo de este estudio fue describir los casos de HA en Galicia en este periodo de brote (PB), compararlos con el periodo pre-brote (PPB), y describir las intervenciones realizadas.

Métodos: Se realizó un estudio descriptivo de los casos de HA declarados entre 2016-2018 (PB), comparados con los del periodo previo (20102015, PPB). Se incluyeron los casos del Sistema de Notificación Obligatoria (por atención primaria, hospitalaria y microbiología) de 2010 a 2018. Se calculó el canal epidémico para el PPB, como media de casos/cuatrisemana para comparar casos observados/esperados. La incidencia (I) [casos por cada 100.000 habitantes $\left.\left(\mathrm{c} / 10^{5} \mathrm{~h}\right)\right]$ por sexo y edad se comparó con el PPB mediante el Riesgo Relativo (RR). Se enviaron mensajes con recomendaciones específicas a través de webs de referencia para hombres que tenían sexo con hombres (HSH).

Resultados: El brote duró 20 cuatrisemanas (septiembre de 2016 a marzo de 2018). La incidencia fue de 3 casos por cada 100.000 habitantes en hombres y 0,5 casos por cada 100.000 habitantes en mujeres. Frente al PPB, el RR-PB en hombres fue $4,8($ IC95\%=4-7) y 20,4 (IC95\%=5-87) entre 40 y 44 años. El $42 \%$ de los hombres respondieron tener relaciones con otros hombres (el $57 \%$ entre 20 y 30 años). A finales de 2016 se envió a través de Wapo (una de las webs de referencia de HSH) un mensaje con recomendaciones (fundamentalmente sobre vacunación), registrándose 331 entradas.

Conclusiones: La incidencia de HA aumenta en Galicia en el período 2016-2018 por un brote en HSH. La susceptibilidad se incrementa entre jóvenes, lo que hace necesario insistir en la vacunación de los grupos de riesgo.

Palabras claves: Brote viral, Hepatitis A, Enfermedades infecciosas, Estudio epidemiológico.

\section{ABSTRACT \\ Hepatitis A outbreak in Galicia during 2016-2018}

Background: In Galicia, the incidence (I) of hepatitis A (HA) is low and the susceptibility is $51 \%$ in adults (18-64 years). Between 2016 and 2018 the cases increased, mainly in men. We intend to describe the cases of HA in Galicia during this outbreak period (PB), compare them with the pre-outbreak period (PPB), and the interventions performed.

Methods: Descriptive study of the cases of HA declared between 2016-18 (PB), compared to those from the previous period (2010-2015, PPB). Cases recorded in the mandatory notification system (general practice, hospitalization and microbiology) from 2010 to 2018 were included. For the pre-outbreak period 2010-2015 (PPB) it was calculated the average of cases/four-week period to compare observed/expected cases; the incidence (I) [cases/100,000 inhabitants $\left.\left(\mathrm{c} / 10^{5} \mathrm{~h}\right)\right]$ by sex and age was compared with the PPB through the Relative Risk (RR). It were sent messages with recommendations through men who have sex with men (MSM) reference websites.

Results: The outbreak lasted 80 weeks (september of 2016 to march of 2018). The incidence was 3 cases $/ 10^{5} \mathrm{~h}$ in men and 0.5 cases $/ 105 \mathrm{~h}$ in women. Compared to the PPB, the RR-PB in men was $4.8(95 \% \mathrm{CI}=4-7)$ and $20.4(95 \% \mathrm{CI}=5-87)$ in $40-44$ years. $42 \%$ of men declared to have relationships with other men (57\% in $20-30$ years). At the end of 2016, a message with recommendations (specially vaccination) was sent via Wapo (promoted to MSM through one of its reference websites), where 331 entries were registered.

Conclusions: HA's incidence, in Galicia, increased in 2016-2018 by an outbreak in MSM. We found an increased susceptibility among young people which makes necessary to insist on the vaccination of groups at risk.

Key words: Outbreaks, Hepatitis A, Infectious disease outbreaks, Epidemiological study. 


\section{INTRODUCCIÓN}

La hepatitis A (HA) es una infección aguda del hígado causada por un virus hepatotrópico no envuelto, de la familia Picornaviridae. El virus se replica en los hepatocitos y se libera en la secrección biliar, encontrándose en altas concentraciones en las heces ${ }^{(1)}$. El periodo de incubación es de 15 a 50 días.

El mecanismo de transmisión principal es feco-oral, a través del consumo de agua o comida contaminada, o por el contacto directo persona a persona, especialmente a través de las relaciones sexuales oro-anales ${ }^{(2)}$. La transmisión es mayor desde dos semanas antes hasta una semana después de la aparición de la ictericia o de la máxima actividad de las enzimas hepáticas.

Existen formas asintomáticas, subclínicas y clínicas con ictericia y coluria ${ }^{(3)}$. La gravedad aumenta con la edad. La insuficiencia hepática fulminante y la muerte son raras, y más frecuentes entre personas de mayor edad o con enfermedad hepática subyacente.

Las principales medidas de control son garantizar un adecuado saneamiento y seguridad alimentaria, educar sobre las prácticas de higiene personal y la inmunización pre-exposición de la población de riesgo ${ }^{(4,5)}$.

Se estima que en el mundo se producen 1,5 millones de casos anuales de HA. España es un país de baja endemicidad (entre 2012 y 2015, la incidencia anual fue menor de $1,5 \mathrm{c} / 10^{5} \mathrm{~h}$ (casos notificados por cada 100.000 habitantes) (1). En Galicia, entre 2000 y 2007 la incidencia anual se mantuvo por debajo de $1 \mathrm{c} / 10^{5} \mathrm{~h}$, hasta que en el año 2008 se produjo un brote de HA debido a hombres que tienen sexo con hombres (HSH) en la provincia de Pontevedra ${ }^{(6)}$.

La última encuesta de seroprevalencia realizada en Galicia en 2013 (entre adultos de
18-64 años) evidenció una pérdida de inmunidad frente al virus de HA (VHA), registrándose un $30 \%$ de susceptibles en 2001 , frente al $50,5 \%{ }^{(7)}$ en 2013. Por grupos de edad, la prevalencia de susceptibles fue del $90,7 \%$ entre los de 18-24 años, del 84,9\% entre los de 25-29 años y del 67,1\% entre los de 30-39 años.

En este contexto, en el verano de 2016 varios países, entre ellos España, declararon un aumento de casos de HA entre $\mathrm{HSH}^{(8)}$, también observado en Galicia. El objetivo de este trabajo fue describir los casos de HA producidos en Galicia durante el brote, compararlo con el periodo prebrote y describir las intervenciones realizadas.

\section{SUJETOS Y MÉTODOS}

Diseño y período de estudio: Se realizó la descripción de los casos de HA declarados entre 2016-2018 (período del brote), comparado con los del periodo previo (2010-2015, período prebrote). Se consideró como final del brote la cuatrisemana 12/2018 para asegurar su finalización, y como inicio del período pre-brote el año 2010 , porque en 2008-09 hubo otro brote de HA.

Definición del caso: Se buscaron pacientes con clínica compatible y serología (IgM anti-VHA) positiva. Cada caso se asignó a una semana epidemiológica, según la fecha de inicio de los síntomas.

Fuentes de datos: En Galicia, la notificación de HA (enfermedad de declaración obligatoria) se realiza por captura automática de los casos registrados en la historia clínica en atención primaria, y de todas las IgM anti-VHA positivas identificadas en los servicios de microbiología de todos los hospitales públicos de Galicia, a través del SIMGA (Sistema de Información Microbiológica de Galicia). Esta información se cruzó para eliminar duplicados.

Además, desde 2008 se recoge una encuesta con los principales factores de riesgo (tabla 3 ), 
entre los que figura el sexo, si se mantuvieron relaciones sexuales en las 8 semanas previas al inicio de los síntomas, y si esas relaciones fueron con hombres.

Esta información se completó con el CMBDAH (Conjunto Mínimo Básico de Datos de Altas Hospitalarias) de los ingresos por HA aguda, con los códigos CIE-9-MC (070, hepatitis vírica; 070.0, hepatitis viral A con coma hepático; 070.1, hepatitis viral A sin coma hepático) y CIE-10-ES (B15, hepatitis aguda tipo A; B15.0 hepatitis aguda tipo A, con coma hepático; B15.9, hepatitis aguda tipo A, sin coma hepático).

Para el cálculo de incidencias se utilizó la población del Padrón Municipal de habitantes, y el número de vacunas administradas frente a VHA o VHA+B se obtuvo del registro del Programa Gallego de Vacunación del Servicio de Enfermedades Transmisibles de la Dirección Xeral de Saúde Pública (DXSP) de Galicia.

Intervenciones realizadas: Se promovió la vacuna frente al VHA y se proporcionó información directa del brote a HSH a través de una de sus webs de referencia (Wapo), donde se publicaron tres campañas. Así, en noviembre de 2016, junio de 2017 y mayo de 2018, se comentó la evolución del brote y se promovieron el sexo seguro y la vacunación. Estas campañas se vincularon a la página web de la DXSP, con información más específica ${ }^{(2)}$.

Dada la situación de desabastecimiento de la vacuna entre junio de 2017 y mayo de 2018, se insistió, tanto por Venres Epidemiolóxico (publicación de la DXSP enviada por correo electrónico a todos los médicos de atención primaria del Servicio Galego de Saúde) como a través de una instrucción específica enviada a cada área sanitaria, sobre la priorización del uso de la vacuna entre HSH.
Análisis estadístico: Se calculó el canal epidémico para el período 2010-2015, empleando la media de los casos para la misma cuatrisemana de los 5 años previos. También se obtuvieron las incidencias por sexo, edad y provincia, así como los riesgos relativos (RR) por sexo ( $R R$ de ser hombre en cada período [RRH], hombres frente a mujeres), con intervalo de confianza con un nivel de significación del 95\%, e igualmente los RR entre el período del brote y el período anterior. Para describir el impacto de la primera campaña en Wapo, se consultó el "número de páginas vistas únicas" (entradas) y el "promedio de tiempo en la página" (PTP) a través de la aplicación Google Analytics para la página web de la DXSP (informe interno). Se solicitó a Wapo el número de mensajes emitidos sobre HA y de consultas recibidas en el perfil institucional.

Para el análisis de datos, se utilizó Excel 2007 y Epidat 4.2.

\section{RESULTADOS}

El brote tuvo lugar entre las cuatrisemanas 09/2016 y 03/2018, con un total de 150 casos declarados, una ratio hombre/mujer de 5/1, y una incidencia casi 4 veces mayor que la observada en el periodo pre-brote.

Descripción temporal: En la cuatrisemana epidemiológica 9/2016 se produjo un aumento de los casos observados sobre los esperados (figura 1), iniciándose un periodo con incidencias iguales o superiores a $0,1 \mathrm{c} / 10^{5} \mathrm{~h}$ (percentil 99 de la serie de incidencias cuatrisemanales de la cuatrisemana $1 / 2010$ a la 13/2015). Esta tendencia se mantuvo hasta la cuatrisemana 2/2018, descendiendo los casos observados por debajo de los esperados en la $3^{\text {a }}$ cuatrisemana.

El brote tuvo una duración de 20 cuatrisemanas, con mayor intensidad en las cuatrisemanas 
Figura 1

Número cuatrisemanal de casos de hepatitis A notificados en Galicia superpuestos al canal epidémico del periodo 2010-2015 con su intervalo de confianza al $95 \%$.

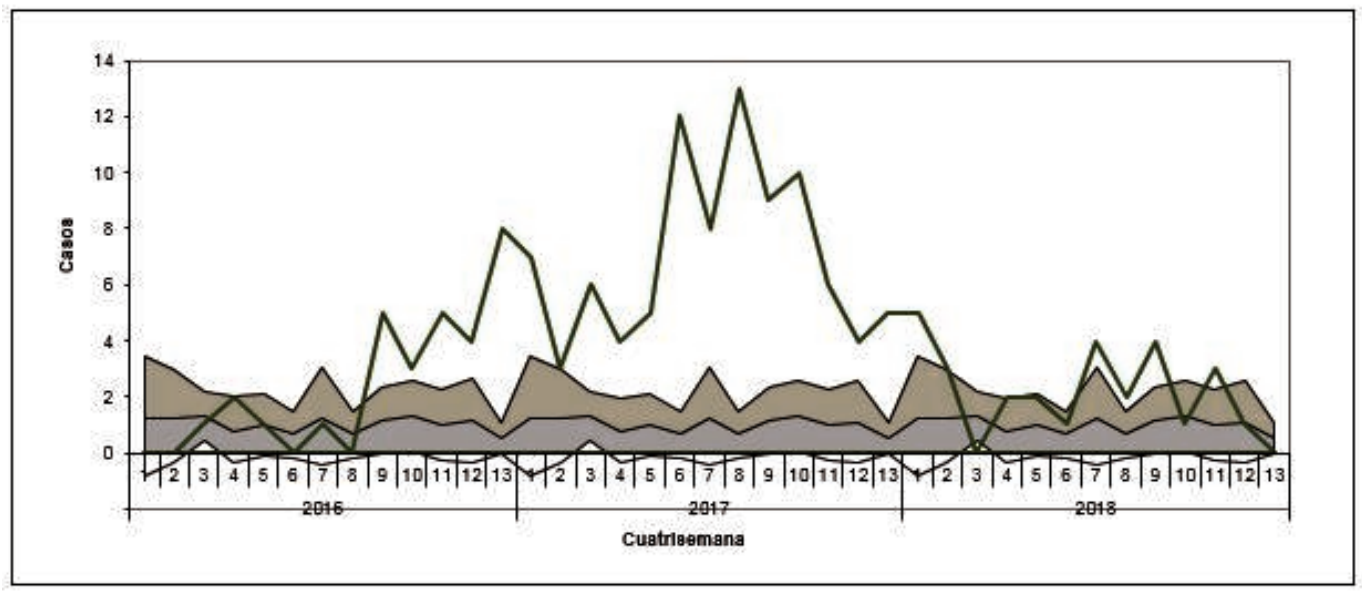

$13 / 2016,6 / 2017$ y 8/2017. Se observaron dos repuntes en las cuatrisemanas $7 / 2018$ y $9 / 2018$.

Descripción geográfica: Se observó un aumento de casos en todas las provincias (figura 2). La mayor incidencia se detectó en la provincia de A Coruña, con $4,1 \mathrm{c} / 10^{5} \mathrm{~h}$ y la menor en Ourense, con $2,6 \mathrm{c} / 10^{5} \mathrm{~h}$. La comparación de las incidencias se recoge en la tabla 1 .

Descripción de los casos: Por sexo y edad, desde 2010 la incidencia de HA fue siempre mayor

\section{Figura 2}

Incidencias anuales de hepatitis A por $10^{5} \mathrm{~h}$, por provincias y el total de Galicia.

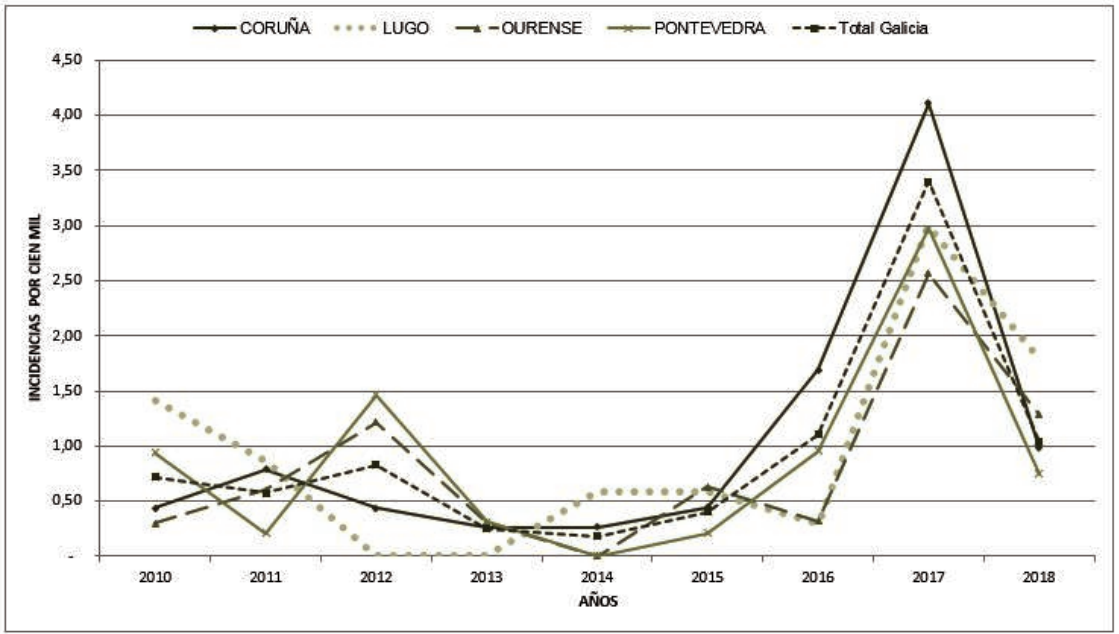




\section{Tabla 1}

Incidencia anual (casos por 100.000 habitantes) por provincias y total de Galicia y riesgo relativo en el periodo de brote frente al periodo anterior (RR 16-18 vs RR 10-15) con su intervalo de confianza al $95 \%$.

\begin{tabular}{|c|c|c|c|c|}
\hline $\begin{array}{c}\text { Incidencia } \\
\text { por } 10^{5} \text { h año }\end{array}$ & $\begin{array}{c}2010- \\
2015\end{array}$ & $\begin{array}{c}2016- \\
2018\end{array}$ & $\begin{array}{c}\text { RR } \\
16-18 \\
\text { frente } \\
\text { a 10-15 }\end{array}$ & IC 95\% \\
\hline A Coruña & 0,4 & 2,2 & 5,1 & $3,3-8,1$ \\
\hline Lugo & 0,5 & 1,6 & 2,9 & $1,3-6,7$ \\
\hline Ourense & 0,5 & 1,3 & 2,7 & $1,1-6,9$ \\
\hline Pontevedra & 0,5 & 1,5 & 2,9 & $1,8-4,9$ \\
\hline Galicia & 0,4 & 1,8 & 3,7 & $2,8-4,9$ \\
\hline
\end{tabular}

en hombres (RRH 2010-2015=1,9), acentuándose durante el brote (RRH 2016-2018=5,3) (ver tabla 2).

El incremento de las incidencias totales observadas durante 2016-2018 fue, prácticamente, a expensas del aumento en hombres entre la $2^{\mathrm{a}}$ y $4^{\mathrm{a}}$ década de la vida (figura 3 ). Entre los hombres se observó un aumento de la incidencia, estadísticamente significativo, en todos los grupos de edad excepto entre 0-14 y los mayores de 60 años (tabla 2). La incidencia entre hombres prácticamente se quintuplicó durante el brote respecto al período previo $(\mathrm{R}=4,8)$.

En mujeres no se observaron aumentos estadísticamente significativos en ningún grupo

\begin{tabular}{|c|c|c|c|c|c|c|c|c|c|c|}
\hline \multicolumn{10}{|c|}{$\begin{array}{c}\text { Tabla 2. } \\
\text { Incidencia anual (c/10 } \mathbf{5} \text { h) en hombres (H) y mujeres (M) en Galicia }\end{array}$} \\
$\begin{array}{c}\text { en el período 2010-2015 y en el trienio 2016-2018, riesgo refativo (RR) de ser hombre } \\
\text { en cada periodo y riesgo relativo en el periodo de brote frente a periodo anterior } \\
\text { (RR 16-18 vs RR 10-15) en hombres y mujeres con sus IC 95\%, por grupo de edad. }\end{array}$ \\
\hline $\begin{array}{c}\text { Edad } \\
\text { (años) }\end{array}$ & \multicolumn{2}{|c|}{$2010-2015$} & \multicolumn{3}{|c|}{$2016-2018$} & \multicolumn{3}{c|}{ RR 16-18 frente a 10-15 } \\
\hline & H & M & RRH & H & M & RRH & H & IC 95\% & M & IC 95\% \\
\hline $0-14$ & 1,1 & 0,7 & 1,6 & 1,2 & 1,7 & 0,7 & 1,0 & $0,3-2,6$ & 2,3 & $0,8-6,3$ \\
\hline $15-19$ & 0,5 & 0,3 & 1,8 & 4,3 & 0,6 & 6,6 & 7,3 & $1,5-35,3$ & 2,1 & $0,1-33,7$ \\
\hline $20-24$ & 0,7 & 0,5 & 1,4 & 6,3 & 1,2 & 5,2 & 8,4 & $2,3-30,1$ & 2,3 & $0,3-16,4$ \\
\hline $25-29$ & 1,8 & 0,6 & 2,9 & 8,5 & 1,0 & 8,3 & 4,6 & $2,0-10,4$ & 1,6 & $0,2-9,8$ \\
\hline $30-34$ & 1,1 & 0,3 & 3,4 & 7,3 & 0,8 & 8,9 & 6,6 & $2,7-15,8$ & 2,5 & $0,3-18,0$ \\
\hline $35-39$ & 1,0 & 0,7 & 1,3 & 5,9 & 0,6 & 9,4 & 5,7 & $2,4-13,7$ & 0,8 & $0,1-4,3$ \\
\hline $40-44$ & 0,3 & 0,3 & 1,0 & 6,3 & 0,6 & 10,5 & 20,4 & $4,7-87,0$ & 1,9 & $0,2-13,8$ \\
\hline $45-49$ & 0,6 & 0,01 & - & 4,4 & 0,9 & 4,7 & 6,8 & $2,2-20,6$ & - & - \\
\hline $50-59$ & 0,1 & 0,1 & 1,0 & 1,5 & 0,5 & 3,1 & 8,7 & $1,8-40,2$ & 2,8 & $0,4-17,1$ \\
\hline $60+$ & 0,2 & 0,1 & 1,3 & 0,2 & 0,01 & - & 1,1 & $0,2-4,8$ & 0,01 & - \\
\hline Todos & 0,6 & 0,3 & 1,9 & 3,1 & 0,5 & 5,3 & 4,8 & $3,5-6,6$ & 1,7 & $1,0-3,0$ \\
\hline
\end{tabular}




\section{Figura 3}

Incidencias anuales $\left(\mathrm{c} / 10^{5} \mathrm{~h}\right)$ en hombres $(\mathrm{H})$ y mujeres $(\mathrm{M})$ en el periodo pre-brote 2010-2015 y en el periodo del brote 2016-2018, por grupo de edad.

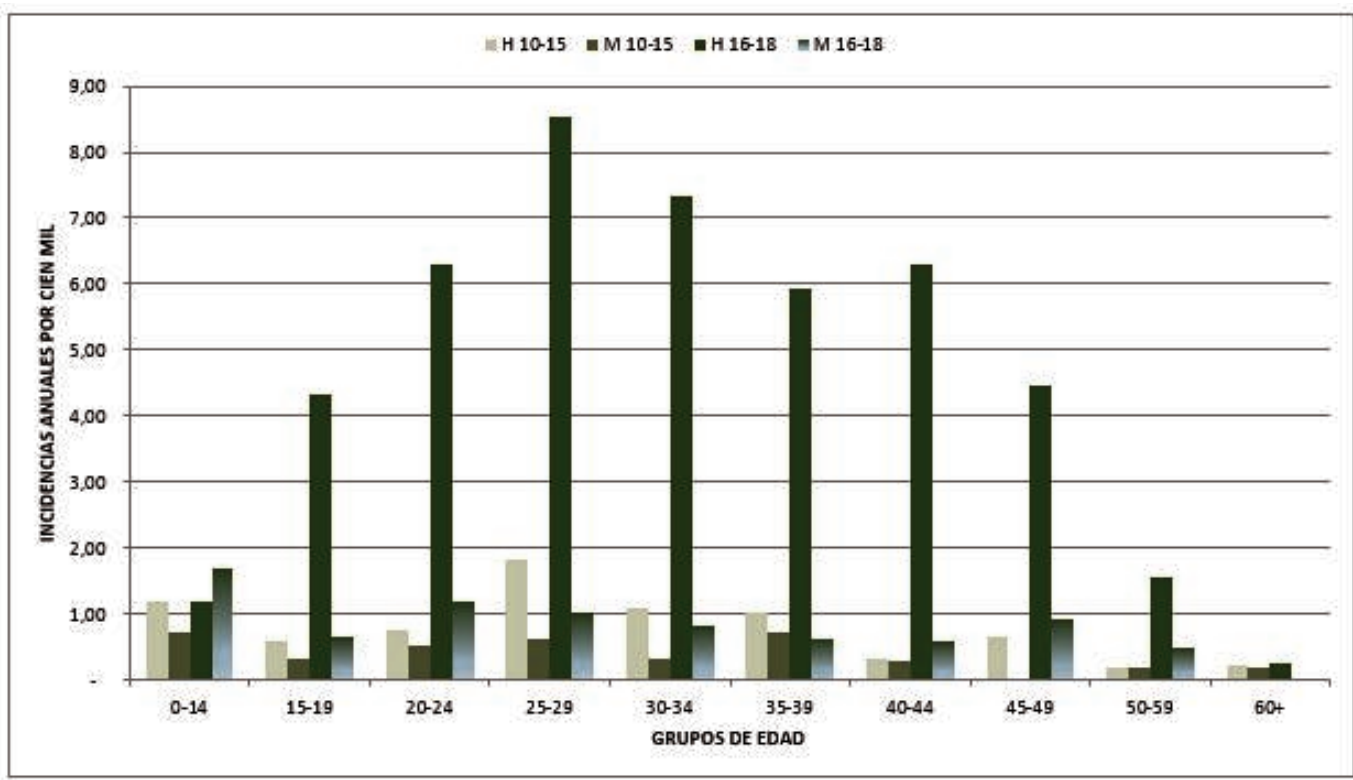

de edad, aunque la incidencia aumentó un 70\% $(\mathrm{RR}=1,7)$ en el brote.

Clínica: El 78\% de los casos tuvieron ictericia, y el 53\% elevación de transaminasas. La mediana de días entre el inicio de síntomas y la confirmación diagnóstica fue de 7 días. El 58\% de los hombres y el $32 \%$ de las mujeres ingresaron.

Factores de riesgo: Al comparar ambos períodos, solo se encontraron diferencias en las variables "contacto sexual con otro caso de HA conocido" (7\% en 2016-2018 frente a $0 \%$ en 2010-2015) y en "relaciones oro-anales" (33\% en 2016-2018 frente a un 1\% en 2010-2015) (tabla 3). El país al que se viajó con más frecuencia en ambos períodos fue Marruecos.

En el período 2016-2018, 53 hombres respondieron haber mantenido relaciones sexuales con otros hombres. Esto supuso un

\section{Tabla 3}

Factores de riesgo referidos a las 8 semanas previas a la encuesta. Números absolutos y porcentajes del período de brote 2016-2018 y el período previo 2010-2015.

\begin{tabular}{|c|c|c|c|}
\hline \multicolumn{2}{|c|}{ Factores } & $2010-2015$ & 2016-2018 \\
\hline \multicolumn{2}{|c|}{$\begin{array}{l}\text { Factores de riesgo (referidos } \\
\text { a las } 8 \text { semanas previas) }\end{array}$} & 82 casos & 150 casos \\
\hline \multicolumn{2}{|c|}{$\begin{array}{l}\text { Viaje a país de } \\
\text { alta-media endemicidad }\end{array}$} & $13(16 \%)$ & $22(15 \%)$ \\
\hline \multicolumn{2}{|c|}{$\begin{array}{l}\text { Consumo de moluscos } \\
\text { bivalvos crudos }\end{array}$} & $14(17 \%)$ & $20(13 \%)$ \\
\hline \multicolumn{2}{|c|}{ Consumo de verduras crudas } & $41(50 \%)$ & $87(58 \%)$ \\
\hline \multicolumn{2}{|c|}{ Consumo de agua no tratada } & $19(23 \%)$ & $56(37 \%)$ \\
\hline \multirow{2}{*}{$\begin{array}{l}\text { Contacto con } \\
\text { otro caso }\end{array}$} & Sexual & $0(0 \%)$ & $11(7 \%)$ \\
\hline & Familiar & $5(6 \%)$ & $9(6 \%)$ \\
\hline \multicolumn{2}{|c|}{ Relaciones oro-anales } & $1(1 \%)$ & $49(33 \%)$ \\
\hline \multicolumn{2}{|l|}{$\mathrm{HSH}$} & $1(1 \%)$ & $53(35 \%)$ \\
\hline
\end{tabular}


$42,4 \%$ de los hombres y un $35,3 \%$ del total de casos. En el grupo de edad entre 20 y 30 años, este porcentaje de HSH ascendió al 57\% de los hombres. En el período previo de 2010-2015, solo 1 contestó afirmativamente a esta pregunta (1,2\% de los 82 casos notificados). En cuanto al número de parejas sexuales durante el brote, el $54 \%$ tuvo una pareja (no estable en el $41 \%$ ), el $23 \%$ afirmó que dos, el 15\% indicó que tres y el 8\% manifestó cuatro o más. Se observó una evolución paralela en la distribución del total de casos y en los que respondieron ser $\mathrm{HSH}$ (figura 4).

Intervenciones: El 24 de noviembre de 2016 (semana 47/2016) se envió a 37.866 perfiles en Wapo el mensaje recogido en la figura 7. La página sobre HA registró 331 entradas en noviembre y 63 en diciembre, con un promedio de tiempo en la página (PTP) de 2 minutos y 20 segundos y de 1 minuto y 21 segundos, respectivamente. Las estadísticas de acceso a la URL de HA de la web de la Consellería de Sanidade mostraron que apenas hubo accesos registrados en los días anteriores al anuncio en Wapo, y descendieron los hits en los días posteriores, para volver a los valores habituales. Como referencia, en 2016 se registraron en la web 15.804 entradas, con una media diaria de 66 entradas. A través de la web Wapo se recibieron entre 100-150 comentarios y varias consultas, fundamentalmente sobre dónde hacerse el diagnóstico de HA y de VIH, y sobre dónde vacunarse de HA. También se usó la plataforma Wapo para hacer campaña de promoción de la vacuna entre HSH, en las semanas 25-26/2017 y $19-20 / 2018$.

La administración de vacunas frente al VHA (figura 5) aumentó en el tiempo, mientras que en el caso de la vacuna combinada $(\mathrm{VHA}+\mathrm{B})$ se estancó desde 2013.

Figura 4

Distribución del total de casos declarados y los casos en hombres que tuvieron relaciones sexuales con otros hombres (HSH), por cuatrisemanas-año.

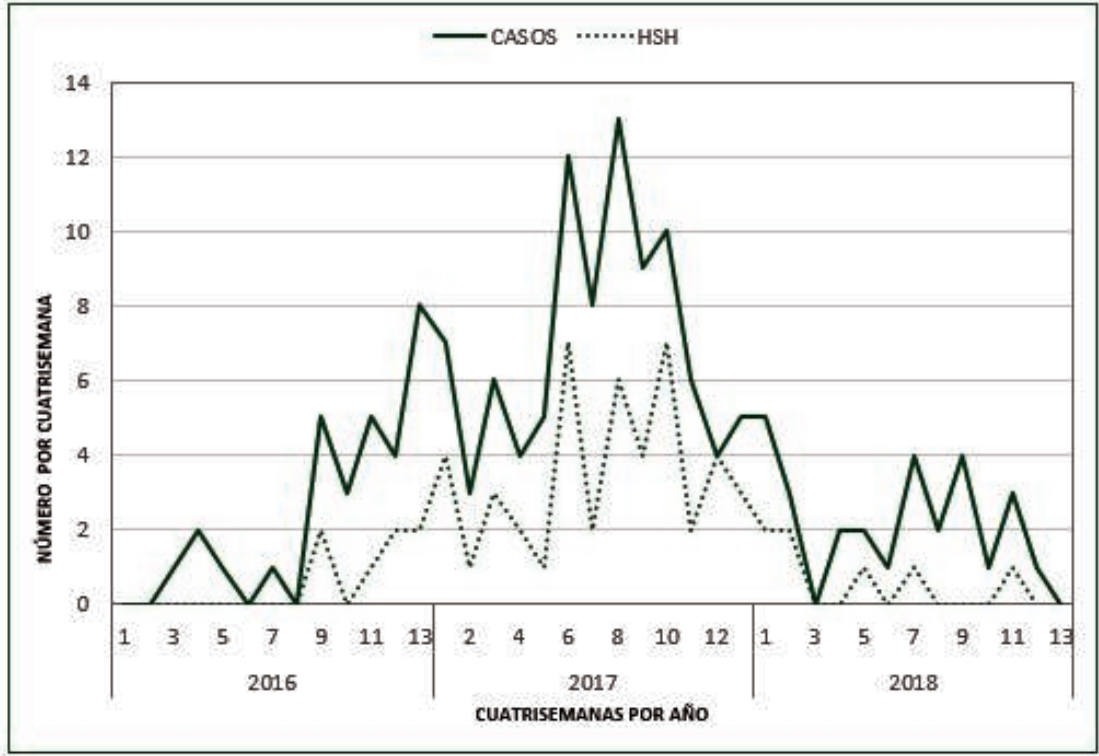


Figura 5

Número de personas vacunadas por cuatrisemana de hepatitis $\mathbf{A} 0 \mathbf{A}+\mathbf{B}$, por sexo (solo una entrada por persona, la de la $1^{\text {a }}$ fecha de administración). Datos apilados.

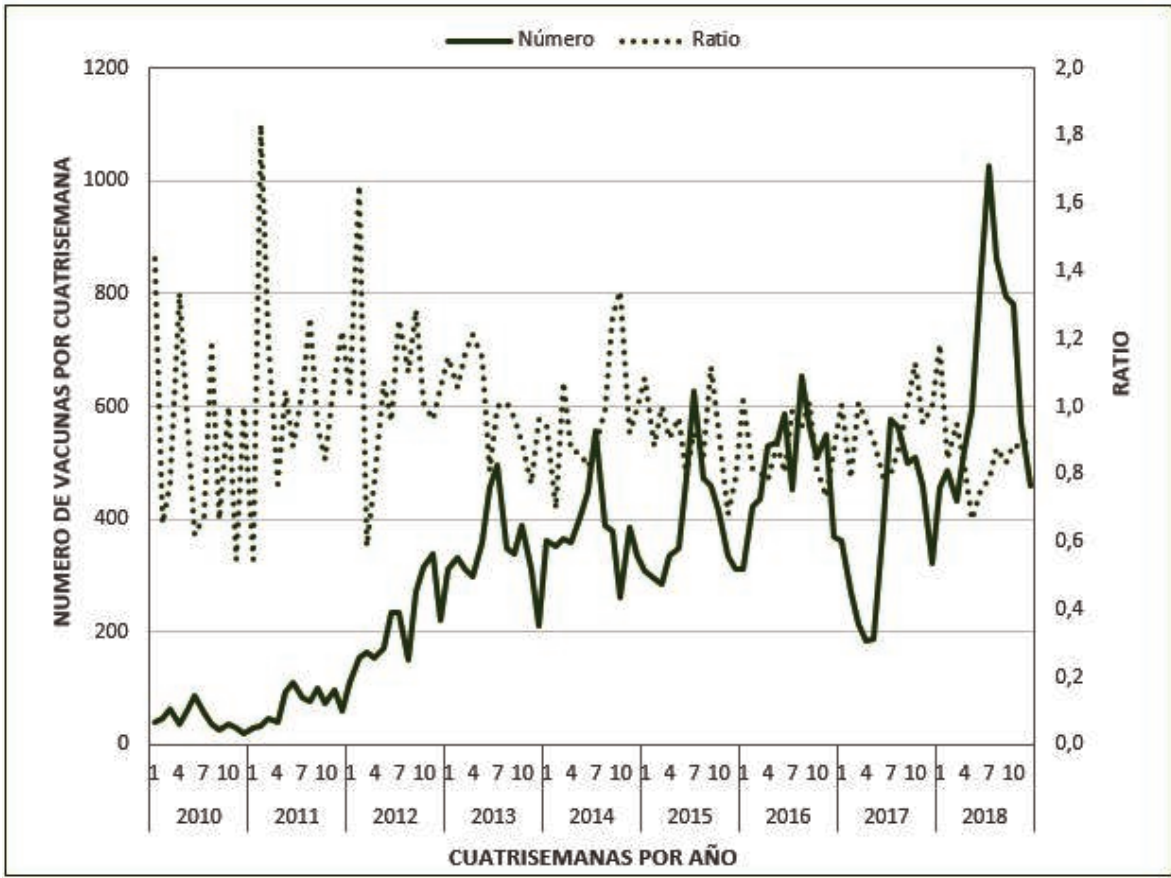

La distribución de vacunas presentó un patrón anual similar, con un pico en los meses previos al verano (junio-julio) y un descenso posterior, con repunte en enero-febrero y septiembre-octubre. Se observaron dos excepciones: en julio de 2016, donde el número de vacunas descendió respecto a los meses anteriores; y a principios de 2017, con un descenso en el número de vacunas administradas, especialmente vacuna frente a HA, y cierto reemplazamiento por vacuna combinada.

En todos los años, excepto 2012, se vacunaron más mujeres que hombres. Por grupos de edad, se observó un descenso en la vacunación del grupo de 0-14 años y un aumento entre los 20-39 años. La ratio de vacunas administradas hombre/mujer a lo largo del tiempo, mostró un aumento desde la cuatrisemana 10/2017 a la cuatrisemana 04/2018 (figura 6).

Análisis filogenético: Se enviaron tres cepas positivas de VHA al Centro Nacional de Microbiología. Una dio negativo y se hizo el estudio filogenético de otra, con resultado de VRD 521 (secuencia UK). 


\section{Figura 6}

Número de vacunas administradas en hombres $(\mathrm{H})$ y mujeres $(\mathrm{M})$, por cuatrisemana, en el periodo pre-brote 2010-2015 y en el periodo del brote 2016-2018.

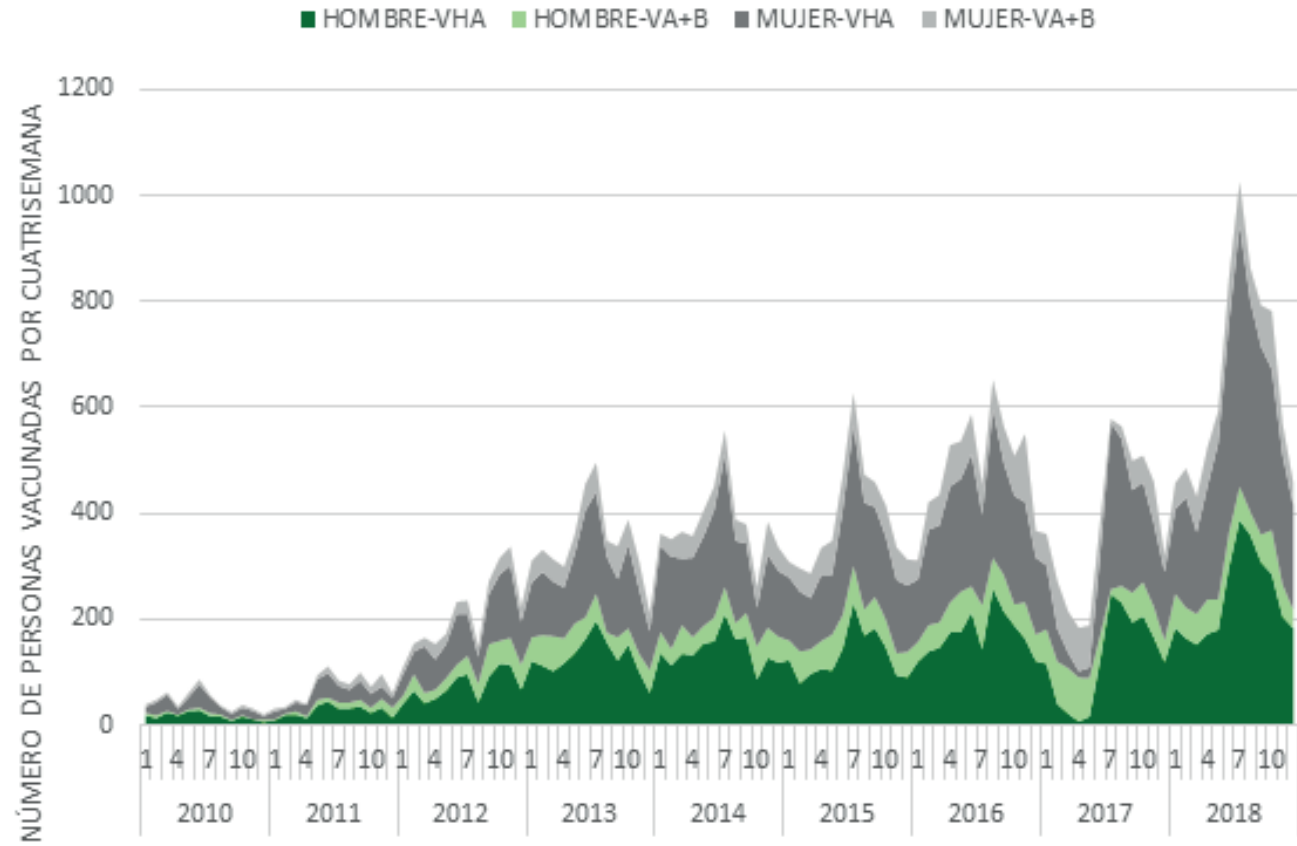

CUATRISEMANA POR AÑO

\section{Figura 7}

Intervenciones.

El 24 de noviembre de 2016 (semana 47/2016) se envió a 37.866 perfiles en Wapo el siguiente mensaje:

Wapo y la Consellería de Sanidade informan de que están a aumentar los casos de hepatitis A en Galicia por transmisión sexual oro-genital y oro-anal. Ten sexo seguro, protégete.

Más información aquí [página sobre hepatitis A de la DXSP: https://www.sergas.es/Saude-publica/ Hepatite--A]; o se puede consultar cualquier duda a través del perfil de Wapo, o llamar al 881542914 [teléfono de contacto de VIH/SIDA, en la DXSP] o escribir a plan.vih.its@sergas.es 


\section{DISCUSIÓN}

En la cuatrisemana 9/2016 se observa en Galicia un aumento de casos de HA respecto al período 2010-2015, que se mantiene hasta el año 2018 y llega a casi cuadriplicar la incidencia, coincidiendo con el brote de HA declarado en Europa entre HSH. Entre junio de 2016 y septiembre de 2018, 24 países declaran 25.032 casos de $\mathrm{HA}^{(9)}$. En España, desde la semana 26/2016 a la semana 48/2018, el número asciende a $7.328^{(10)}$.

Atribuir a cada caso el mecanismo de transmisión es complicado, pero las características del brote y el análisis de exposición a factores de riesgo permiten establecer hipótesis.

Este brote presenta una duración larga y no está concentrado geográficamente, apareciendo casos en todas las provincias gallegas. La extensión, tanto temporal como geográfica, descarta una fuente común y localizada, y orienta hacia una transmisión de fuente continua (alimentaria, por ejemplo) o una transmisión persona a persona. El $75 \%$ de los casos se encuentran entre los 20-40 años y existe un claro predominio de hombres, lo que descarta la hipótesis de fuente alimentaria ya que, en general, se esperaría que se afectasen ambos sexos y todas las edades, teniendo en cuenta los patrones culturales de consumo de alimentos propio de nuestra comunidad.

Al comparar la información recogida del brote con la del período pre-brote, solo se encuentran diferencias, en cuanto a los factores de riesgo, en el contacto sexual con otro caso y en el mantenimiento de relaciones oro-anales. El porcentaje de HSH entre los casos pasa de un $1,2 \%$ en el período pre-brote a un $35,3 \%$ en el brote.

Basándose en la información de la encuesta sobre hábitos sexuales realizada en Galicia durante 2009 (datos no publicados), en la que el
$1,3 \%$ de los hombres respondieron ser $\mathrm{HSH}$, se esperaría encontrar $2 \mathrm{HSH}$ entre los 125 casos varones del brote. Al ser una pregunta con cierta sensibilidad social, podría estar subestimada. Según la encuesta realizada por la empresa alemana Dalia Research, el 6,6\% de los hombres españoles se declararon LGTB (lesbianas, gays, bisexuales, transgénero) ${ }^{(11)}$. Asumiendo este porcentaje, en este brote se esperaría encontrar 8 casos y no los 53 casos declarados como HSH.

Si se considera este porcentaje del $6,6 \%$ como de HSH, en la población de Galicia entre 2016-2018 hay 258.627 HSH. La incidencia en este brote para este colectivo es de $20,5 \mathrm{c} / 10^{5}$ $\mathrm{HSH}$, que, comparado con la incidencia calculada para el total de la población $\left(1,8 \mathrm{c} / 10^{5} \mathrm{~h}\right)$, permite observar que existe 11 veces más riesgo de HA en HSH que en población general, apoyando que la transmisión entre los HSH es primordial para el desarrollo del brote.

La alta tasa de susceptibilidad al VHA es clave para entender la ampliación del brote, que en marzo de 2018 estaba volviendo a su nivel basal, tanto en Galicia como en el resto de Europa ${ }^{(12)}$. Se observan dos repuntes, tanto en la cuatrisemana 7/2018, con tres casos (con factores de riesgo de antecedente de viaje, así como de consumir bivalvos y agua no tratada), como en la cuatrisemana 9/2018, con tres casos intrafamiliares con antecedente de viaje a Marruecos, no incluidos en este brote.

La información sobre la epidemiología molecular de las cepas (VRD 521, secuencia UK) coincide con la cepa más frecuentemente aislada en el brote en Europa. Así, de los 25.032 casos declarados hasta el 14 de septiembre de 2018 en Europa, 4.475 se relacionan mediante epidemiología molecular al presentar una de las tres cepas asociadas al brote ${ }^{(13)}$ : VRD_521_2016, V16-25801, y RIVM-HAV16-090. Aunque la distribución de las cepas varía con el tiempo 
y el país, no se aprecian diferencias en las características de la exposición. Un cuarto de los casos confirmados hasta marzo de 2017 viajan, en el período de incubación, fuera del país declarante. El país más visitado es España ${ }^{(8)}$ donde, a junio de 2017, el análisis filogenético de las cepas aisladas es también mayoritariamente VRD_521_2016 $6^{(1)}$.

Los datos epidemiológicos y de secuenciación facilitan la investigación ${ }^{(14)}$, y permiten confirmar la extensión del brote a población no $\mathrm{HSH}$, lo que explica la presencia de casos de mujeres en el brote y el que varios países declararan pequeños brotes transmitidos por alimentos asociados con estas cepas ${ }^{(8)}$. En Portugal se identifica un clúster entre población romani ${ }^{(15)}$. En Holanda, aproximadamente un tercio de los casos no tienen vínculo $\mathrm{HSH}^{(14)}$. Y en Italia, la inclusión tardía de mujeres en el clúster de la cepa VRD_521_2016 sugiere rutas de transmisión no limitadas a las prácticas sexuales ${ }^{(16)}$.

Como medida de control principal se recomendó la vacunación ${ }^{(12)}$. La administración de vacunas frente al VHA presenta en Galicia una tendencia ascendente, que podría reflejar una mejora del sistema de registro o un aumento en el número de viajes, cada vez más habitual ${ }^{(17)}$. De hecho, el viaje a un país de alta-media endemicidad de HA se cree que es el motivo más frecuente de vacunación ${ }^{(18)}$. Además, explicaría la distribución observada, con picos en los meses previos a las principales épocas de vacaciones. Esta tendencia se interrumpe en 2017 por el desabastecimiento en el suministro por parte de los laboratorios farmacéuticos ${ }^{(19,20,21)}$. Este problema (restablecido en mayo) ${ }^{(22)}$ lleva a difundir recomendaciones de priorización de las dosis existentes. Se considera prioritaria la vacunación a $\mathrm{HSH}$, con la recomendación de indicar una sola dosis durante el desabastecimiento. Así, considerando el desabastecimiento, los viajes, y la imposibilidad de conocer el motivo de vacunación, observar una relación vacuna/brote es difícil, aunque quizá se pueda intuir gracias al ratio de vacunación hombre/ mujer mayor de 1 observado entre las cuatrisemanas 04/2017 y 10/2017. Se administran más vacunas a mujeres, pero esto podría ser por su vinculación al brote o por viajes, y también al grupo de edad de 20-40 años, el más afectado por el brote pero también el que más viaja ${ }^{(23)}$.

Además de la vacunación, cada vez que se declara un caso de HA en atención primaria salta una alerta recordando la actuación sobre los contactos, para valorar la profilaxis postexposición con vacuna y/o Ig, según una instrucción ${ }^{(24)}$. Así mismo, durante el periodo de brote se difunde información sobre la situación y los grupos prioritarios de vacunación a través de Venres Epidemiolóxico ${ }^{(15,24,25)}$, que es una publicación quincenal, de una página de extensión para facilitar su lectura, editada por la Dirección Xeral de Saúde Pública, y dirigida específicamente, aunque no exclusivamente, a los médicos de atención primaria, a los que se les facilita información de su interés desde un punto de vista epidemiológico.

Otra medida implantada para favorecer el control del brote es la difusión, a través de las plataformas digitales, de la importancia de la higiene personal (lavarse las manos y los genitales antes y después de las relaciones sexuales), y del uso de preservativo y de láminas de látex para el sexo oro-anal. Los datos de la campaña en Wapo indican que había sido de interés.

Las limitaciones de este estudio fueron la posible infradeclaración de $\mathrm{HSH}$ (aunque el porcentaje fue superior al referenciado en España $[42,4 \% \text { frente a } 11,5 \%]^{(1)}$ ), la falta de información sobre los hábitos sexuales de los HSH (que podrían haberse modificado en estos años y facilitar la extensión del brote -se podría valorar incluirlo en la encuesta de conductas de riesgo existente-), y el no disponer de información sobre los contactos de los casos 
(aunque el control post-exposición se promovió siguiendo los protocolos existentes).

Como fortalezas podemos destacar la triple vía de entrada de la información (declaración individualizada, SIMGA y CMBD, que hace prácticamente imposible que algún caso no se detectase), el disponer de un grado elevado de cumplimentación de la información sexual de los casos, la relación de actividades realizadas (que pueden ayudar en futuros brotes), y la disponibilidad de datos de vacunación y de la campaña preventiva realizada en Wapo.

Como conclusión, señalar que la incidencia de HA en Galicia, aumentada entre los años 2016 y 2018 por un brote en HSH, volvió a su nivel basal. Las encuestas de seroprevalencia indican alta susceptibilidad entre los jóvenes, lo que hace probable que se produzcan nuevos brotes. La vacunación de los grupos de riesgo es fundamental como principal medida de prevención.

\section{AGRADECIMIENTOS}

Al servicio de Control de Enfermedades Transmisibles de la Dirección Xeral de Saúde Publica, por facilitar la información relativa a las vacunas frente al VHA administradas durante el período a estudio, así como por la evaluación de la campaña realizada entre los $\mathrm{HSH}$ durante el brote.

\section{BIBLIOGRAFÍA}

1. Centro Nacional de Epidemiología. Situación de la hepatitis A en 2016-2017.CNE; 2017 mar.

2. Hepatitis A - Consellería de Sanidade - Servizo Galego de Saúde [Internet]. [citado 25 de febrero de 2019]. Disponible en: https://www.sergas.es/Saude-publica/Hepatite--A.

3. DXSP. Protocolo de vixilancia e control da hepatite A en Galicia [Internet]. 2010 [citado 25 de febrero de 2019].
Disponible en: https://www.sergas.es/Saude-publica/ Documents/1142/Protocolo\%20Hepatite\%20A.pdf.

4. European Centre for Disease Prevention and Control. Factsheet about hepatitis A [Internet]. [citado 25 de febrero de 2019]. Disponible en: http://ecdc.europa.eu/en/hepatitis$\mathrm{A} /$ facts.

5. Organización Mundial de la Salud. Hepatitis A [Internet]. [citado 25 de febrero de 2019]. Disponible en: https://www. who.int/es/news-room/fact-sheets/detail/hepatitis-a.

6. DXSP. Abrocho de hepatite A en Galicia durante 20082009. BEG. 2011;XXIII(2):15-20.

7. DXSP. Enquisa Galega de seroprevalencia 2013. BEG. 2014;XXVI(4):1-14.

8. Ndumbi P, Freidl GS, Williams CJ, Mårdh O, Varela C, Avellón A et al. Hepatitis A outbreak disproportionately affecting men who have sex with men (MSM) in the European Union and European Economic Area, June 2016 to May 2017. Euro Surveill. 2018;23(33).

9. European Centre for Disease Prevention and Control. Epidemiological update: Hepatitis A outbreak in the EU/ EEA mostly affecting men who have sex with men. Epidemiological update (12 Sep 2018) [Internet]. 2018 [citado 25 de febrero de 2019]. Disponible en: http://ecdc.europa. eu/en/news-events/epidemiological-update-hepatitis-outbreak-eueea-mostly-affecting-men-who-have-sex-men-2.

10. Centro Nacional de Epidemiología. Situación de la hepatitis A (semana 48/2018). Boletín epidemiológico semanal en RED [Internet]. 26 de diciembre de 2018 [citado 26 de febrero de 2019]; Disponible en: http://www.isciii. es/ISCIII/es/contenidos/fd-servicios-cientifico-tecnicos/fdvigilancias-alertas/fd-boletines/fd-boletin-epidemiologicosemanal-red/pdf_2018/IS-181226-WEB.pdf.

11. Counting the LGBT population: 6\% of Europeans identify as LGBT [Internet]. Dalia Research. 2016 [citado 25 de febrero de 2019]. Disponible en: https://daliaresearch.com/counting-the-lgbt-population-6-of-europeans-identify-as-lgbt/. 
12. European Centre for Disease Prevention and Control. Epidemiological update: hepatitis A outbreak in the EU/ EEA mostly affecting men who have sex with men. Epidemiological update (23 Mar 2018) [Internet]. 2018 [citado 25 de febrero de 2019]. Disponible en: http://ecdc.europa.eu/en/news-events/epidemiological-update-hepatitis-outbreak-eueea-mostly-affecting-men-who-have-sex-men-1.

13. European Centre for Disease Prevention and Control. Hepatitis A outbreaks in the EU/EEA mostly affecting men who have sex with men. Rapid Risk Assessment (First update, 23 February 2017) [Internet]. [citado 26 de febrero de 2019]. Disponible en: https://ecdc.europa.eu/sites/portal/files/ media/en/publications/Publications/16-02-2017-RRA\%20 UPDATE\%201-Hepatitis\%20A-United\%20Kingdom.pdf.

14. Friesema IH, Sonder GJ, Petrignani MW, Meiberg AE, Rijckevorsel GG van, Ruijs WL et al. Spillover of a hepatitis A outbreak among men who have sex with men (MSM) to the general population, the Netherlands, 2017. Eurosurveillance. 7 de junio de 2018;23(23):1800265.

15. DXSP. Restablecemento da vacinación fronte a hepatite A. Venres epidemiolóxico [Internet]. 4 de mayo de 2018 [citado 26 de febrero de 2019];7(9). Disponible en: https://www. sergas.es/Saude-publica/Documents/5032/Venres $\% 20$ Epidemioloxico_vol7_n\%C2\%BA9_04052018.pdf.

16. Lanini S, Minosse C, Vairo F, Garbuglia A, Di Bari V, Agresta A et al. A large ongoing outbreak of hepatitis A predominantly affecting young males in Lazio, Italy; August 2016 - March 2017. PLoS ONE. 2017;12(11):e0185428.

17. Organización Mundial del Turismo. Resultados del Turismo Internacional 2018 y Perspectivas 2019 [Internet]. 2019. Disponible en: http://cf.cdn.unwto.org/sites/all/files/ pdf/unwto_barometer_jan19_presentation_sp.pdf.

18. Gossner CM, Severi E, Danielsson N, Hutin Y, Coulombier D. Changing hepatitis A epidemiology in the European Union: new challenges and opportunities. Eurosurveillance. 23 de abril de 2015;20(16):21101.
19. Junta de Andalucía. Instrucciones para la vacunación frente a hepatitis A en adultos [Internet]. 2016 [citado 26 de febrero de 2019]. Disponible en: http://www.juntadeandalucia.es/servicioandaluzdesalud/distritomalaga/docs/cuidados/vacunas/INSTRUCCIONES\%20PARA\%20LA $\% 20$ VACUNACION\%20FRENTE\%20A\%20HEPATITIS $\% 20$ A\%20EN\%20ADULTOS.pdf.

20. Consejo Interterritorial. Problemas de suministro frente a Hepatitis A. Recomendaciones [Internet]. 2017 [citado 26 de febrero de 2019]. Disponible en: https://www.mscbs. gob.es/profesionales/saludPublica/prevPromocion/vacunaciones/docs/Problemas_suministro_HepatitisA.pdf.

21. DXSP. Nota informativa sobre vacinación fronte á hepatite A en adultos [Internet]. 2017 [citado 26 de febrero de 2019]. Disponible en: https://www.sergas.es/Saudepublica/Documents/4490/Vacinaci $\%$ C3\%B3n $\% 20$ fronte $\% 20 \%$ C3\%A1\%20hepatite $\% 20$ A $\% 20$ en $\% 20$ adultos.pdf.

22. DXSP. Nota informativa sobre o restablecemento da vacinación fronte á hepatite A en adultos [Internet]. 2018 [citado 26 de febrero de 2019]. Disponible en: https://www.sergas.es/Saude-publica/Documents/5018/ Restablecemento_HEPATITE-A.pdf.

23. Muñoz-Cidad TM, Mateos-Conde J, González-Pérez LC, Aránzazu García-Iglesias M. Consultas de viajeros en un centro de vacunación internacional. Gac Sanit. 1 de enero de $2014 ; 28(1): 86-7$.

24. DXSP. Situación da Hepatite A en Galicia: A profilaxe. Venres epidemiolóxico [Internet]. 27 de abril de 2012 [citado 26 de febrero de 2019];1(8). Disponible en: https://www. sergas.es/Saude-publica/Documents/1723/vol1_n8.pdf

25. DXSP. Abrocho de hepatite A en HSH: prioridade para a vacinación. Venres epidemiolóxico [Internet]. 7 de septiembre de 2018 [citado 26 de febrero de 2019];7(18). Disponible en: https://www.sergas.es/Saudepublica/Documents/4500/venres_epidemioloxico_vol6_ n12_20170615.pdf. 